\title{
BCG Vaccination Policy and Protection Against COVID-19
}

\author{
Jitendra Meena ${ }^{1} \cdot$ Arushi Yadav $^{2} \cdot$ Jogender Kumar $^{3}$ (I)
}

Received: 18 May 2020 / Accepted: 28 May 2020 / Published online: 9 June 2020

(C) Dr. K C Chaudhuri Foundation 2020

To the Editor: Wide disparity in disease burden and mortality in COVID-19 among countries is intriguing. BCG vaccine is thought to be one of the factors attributing to this discrepancy. To explore this hypothesis, we assessed the correlation of COVID-19 burden at three different time points (14 April, 29 April, and 13 May 2020) with year-wise BCG coverage among the countries with universal BCG vaccination policy over past four decades (1980-2018). Relevant data were extracted and analyzed using SPSS $[1,2]$. Out of 170 countries for which data on BCG vaccination and COVID-19 was available, we excluded 28 countries $(<100$ cases as on May 13,2020$)$. Thirteen $(9.2 \%)$ countries didn't have a universal BCG vaccination policy at any time from 1980 to 2018.

Among the countries with universal BCG vaccination policy (n-142), a weak but positive correlation (spearmen rho- 0.1-0.5, $p<0.05$ ) was observed between COVID-19 cases and deaths per million population and BCG vaccination coverage rates (Supplementary Table 1). There was no significant correlation between case-fatality rate and BCG coverage at any of the abovementioned time points. We further explored the relationship by adjusting for covariates (age $>65 \mathrm{y}$, cardiovascular disease death rate, diabetes, GDP per capita, beds per thousand population, and the number of tests done per million population) and didn't find any consistent and significant relationship between BCG vaccination rates and COVID-19 burden. These results suggest against any meaningful relationship between country's BCG vaccination coverage and COVID-19 burden.

Electronic supplementary material The online version of this article (https://doi.org/10.1007/s12098-020-03371-3) contains supplementary material, which is available to authorized users.

Jogender Kumar

Jogendrayadv@gmail.com

1 Department of Pediatrics, All India Institute of Medical Sciences, New Delhi, India

2 Department of Radiodiagnosis and Imaging, Government Medical College and Hospital, Chandigarh, India

3 Department of Pediatrics, Post Graduate Institute of Medical Education and Research, Chandigarh, India
Preprint studies have reported a negative association between BCG and COVID-19 [3]. However, these associations are spurious as they chose $\mathrm{BCG}$ vaccination as a dichotomous variable (yes/no, counted yes even if the coverage is $5-10 \%$ and vaccination was stopped 2-3 decades back) and didn't adjust for potential confounders [4].

The association between COVID-19 and BCG vaccine is postulated due to the non-specific effects (NSE) inherent to the vaccine. However, these NSE's may not last beyond 5-10 y [4]. A recent study failed to show the protective effect of $\mathrm{BCG}$ over COVID-19 [5].

We acknowledge that these results are based on epidemiological data and have inherent biases, therefore, well-designed clinical trials are needed to test this hypothesis. Existing epidemiological evidence didn't recommend using BCG against COVID-19 in routine clinical practice.

\section{Compliance with Ethical Standards}

Conflict of Interest None.

\section{References}

1. World Health Organization. Coronavirus Disease (COVID-2019) Situation Reports. Available at: https://www.who.int/emergencies/ diseases/novel-coronavirus-2019/situation-reports. Accessed 16 May 2020.

2. World Health Organization. BCG Immunization Coverage Estimates By Country. Global Health Observatory Data Repository. Available at: https://apps.who.int/gho/data/view.main.80500?lang=en. Accessed 15 May 2020.

3. Riccò M, Gualerzi G, Ranzieri S, Bragazzi NL. Stop playing with data: there is no sound evidence that Bacille Calmette-Guérin may avoid SARS-CoV-2 infection (for now). Acta Bio Medica Atenei Parmensis. 2020;91:207-13.

4. Kumar J, Meena J. Demystifying BCG vaccine and COVID-19 relationship. Indian Pediatr. 2020, Apr 30. pii: S097475591600168.

5. SARS-CoV-2 Rates in BCG-Vaccinated and Unvaccinated Young Adults. JAMA. Available at: https://jamanetwork.com/journals/ jama/fullarticle/2766182. Accessed 18 May 2020.

Publisher's Note Springer Nature remains neutral with regard to jurisdictional claims in published maps and institutional affiliations. 